\title{
Infecção hospitalar em Unidade de Terapia Intensiva Neonatal: desvendando "mitos" sobre a influência do local de nascimento
}

\author{
Nosocomial infection in Neonatal Intensive Care Units: disclosing "myths" regarding the influence \\ of the place of birth
}

nfecção hospitalar (IH) em Unidade de Terapia Intensiva Neonatal (UTIN) é sempre um assunto de extrema relevância, especialmente no nosso meio. Não passamos mais de um ano sem ouvirmos, na mídia, relatos dramáticos de surtos e epidemias de $\mathrm{IH}$ em unidades neonatais, invariavelmente relacionados à superlotação das unidades e às deficiências grosseiras de recursos humanos e infraestrutura.

O artigo Infecção hospitalar em Unidade de Terapia Intensiva Neonatal: há influencia do local de nascimento ${ }^{(1)}$ nos faz refletir sobre um aspecto muito interessante relacionado ao risco de aquisição de $\mathrm{IH}$ : o local de nascimento. Sabemos da deficiência de leitos hospitalares para recém-nascidos de alto risco no nosso país e, em decorrência, muitos neonatos acabam internados em unidades pediátricas, com menor experiência no manuseio dessa população de alto risco, o que os leva, portanto, a um risco maior de aquisição de $\mathrm{IH}$ e suas consequências. Outro aspecto é o "mito" de que recém-nascidos oriundos de outros serviços de saúde, ou mesmo do domicílio, trariam maior risco de introdução de germes nosocomiais e aumento das taxas de IH para a UTI. Poucos estudos abordam esse aspecto ${ }^{(2,3)}$, que é de extrema importância, pois teoricamente sabe-se que o melhor local de assistência para tais crianças (internas ou externas) é a UTIN. Assim, muitos serviços de referência neonatal evitam a admissão de pacientes externos de alto risco, tentando "preservar" sua flora e sua taxa de IH. Entretanto, será mesmo verdade que esse risco existe?

$\mathrm{O}$ presente estudo analisou a frequência de aquisição de IH nos recém-nascidos internos e externos, não evidenciando nenhuma diferença estatística entre os grupos. Mesmo analisando separadamente os índices de $\mathrm{IH}$ precoce (provável origem materna e canal de parto) e tardia (provavelmente de origem hospitalar e relacionada à assistência dentro da unidade), os autores não encontraram diferença entre os pacientes externos e aqueles nascidos na própria instituição(1). Tais resultados podem estar associados ao pequeno número de pacientes estudados, mas merecem reflexão por se constituírem em dado de impacto para a mudança de hábitos e conceitos.

Outro resultado importante que o presente estudo nos fornece está relacionado ao tipo de germe prevalente nos recém-nascidos de origem interna e externa, quando ocorre a infecção. Como teoricamente esperado, os de origem externa apresentaram mais infecções por germes ditos "nosocomiais" como a Pseudomonas spp e o Enterobacter spp, comparados aos de origem interna. Porém, não fica claro, na descrição do estudo, se os pacientes internos e externos eram assistidos de forma separada. Na discussão, os autores afirmam que os pacientes externos com mais de 48 horas de vida eram mantidos em precauções de

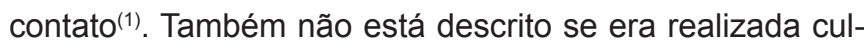
tura de vigilância dos recém-nascidos externos com mais de 48 horas de vida e se havia alguma relação da flora admissional com a flora causadora de $\mathrm{IH}$. De qualquer forma, o resultado mostrado pelos autores reforça a indicação de que é sempre saudável vigiar a flora "externa" à unidade, visto que, mesmo com pouco tempo de exposição, já há diferença de germes causadores de $\mathrm{IH}$. Acredita-se que, sempre que possível, deve-se recomendar que neonatos de origem interna e externa sejam mantidos em coortes de funcionários, em ambientes diferentes e em precaução de contato $^{(4,5)}$.

Atualmente, muito se conhece a respeito da prevenção das IH nos recém-nascidos de alto risco. Apesar disso, vários são os "mitos" criados e tidos como dogmas na nossa prática diária, ainda que sem evidências científicas estabelecidas. Exemplo dessa prática é o uso de luvas estéreis para a manipulação dos neonatos com peso ao nascimento inferior a $1.000 \mathrm{~g}$ até o $15^{\circ}$ dia de vida ${ }^{(6)}$. Não há evidência de benefício de tal prática. Portanto, o presente estudo nos faz rever conceitos relacionados à influência ou não do local de nascimento no desenvolvimento de infecção hospitalar. Isso reforça a importância de uma assistência adequada na UTIN, feita por profissionais habilitados, como um determinante de sucesso.

Rosana Richtman ${ }^{1}$ 


\section{Referências bibliográficas}

1. Pinheiro MB, Nicoletti C, Boszczowski I, Puccini DM, Ramos SR. Infecção hospitalar em Unidade de Terapia Intensiva Neonatal: há influencia do local de nascimento? Rev Paul Pediatr 2009;27:6-14.

2. Dalben M, Varkulja G, Basso M, Krebs VL, Gibelli MA, van der Heijden I et al. Investigation of an outbreak of Enterobacter cloacae in a neonatal unit and review of the literature. J Hosp Infect 2008:70:7-14.

3. Gastmeier P, Loui A, Stamm-Balderjahn S, Hansen S, Zuschneid I, Sohr D et al. Outbreaks in neonatal intensive care units - they are not like others. Am J Infect Control 2007;35:172-6.
4. Pessoa-Silva CL, Richtmann R, Calil R, Santos RM, Costa ML, Frota AC et al. Healthcare-associated infections among neonates in Brazil. Infect Control Hosp Epidemiol 2004;25:772-7.

5. Srivastava S, Shetty N. Healthcare-associated infections in neonatal units: lessons from contrasting worlds. J Hosp Infect 2007;65:292-306

6. Ng PC, Wong HL, Lyon DJ, So KW, Liu F, Lam RK et al. Combined use of alcohol hand rub and gloves reduces the incidence of late onset infection in very low birthweight infants. Arch Dis Child Fetal Neonatal Ed 2004;89:F336-40.
'Doutora em Medicina pela Universidade de Freiburg - Alemanha, médica infectologista do Instituto de Infectologia Emilio Ribas, presidente da Comissão de Controle de Infecção Hospitalar do Hospital e Maternidade Santa Joana e Pro Matre Paulista, diretora da Associação Paulista de Estudos em Controle de Infecção Hospitalar, Diretora da Sociedade Paulista de Infectologia, São Paulo, SP, Brasil
Endereço para correspondência:

Rua do Paraíso , 450 - Paraíso

CEP 04103-080 - São Paulo/SP

E-mail: rrichtmann@uol.com.br

Recebido em: 14/1/08 